Plasma transport in stochastic magnetic field caused by vacuum resonant magnetic perturbations at diverted tokamak edge

G. Park, C. S. Chang, I. Joseph, R. A. Moyer

September 14, 2010

Physics of Plasmas 
This document was prepared as an account of work sponsored by an agency of the United States government. Neither the United States government nor Lawrence Livermore National Security, LLC, nor any of their employees makes any warranty, expressed or implied, or assumes any legal liability or responsibility for the accuracy, completeness, or usefulness of any information, apparatus, product, or process disclosed, or represents that its use would not infringe privately owned rights. Reference herein to any specific commercial product, process, or service by trade name, trademark, manufacturer, or otherwise does not necessarily constitute or imply its endorsement, recommendation, or favoring by the United States government or Lawrence Livermore National Security, LLC. The views and opinions of authors expressed herein do not necessarily state or reflect those of the United States government or Lawrence Livermore National Security, LLC, and shall not be used for advertising or product endorsement purposes. 


\title{
Plasma transport in stochastic magnetic field caused by vacumm resonant magnetic perturbations at diverted tokamak edge
}

\author{
G. $\operatorname{Park}^{a)}{ }^{*}$ C.S. Chang, ${ }^{\dagger}$ I. Joseph ${ }^{b, c)}$, and R.A. Moyer ${ }^{b)}$ \\ a) Courant Institute of Mathematical Sciences, New York University \\ b) University of California at San Diego \\ c) Present address: Lawrence Livermore National Laboratory
}

\begin{abstract}
A kinetic transport simulation for the first $4 \mathrm{~ms}$ of the vacuum resonant magnetic perturbations (RMPs) application has been performed for the first time in realistic diveted DIII-D tokamak geometry [J. Luxon, Nucl. Fusion 42, 614 (2002)], with the self-consistent evaluation of the radial electric field and the plasma rotation. It is found that, due to the kinetic effects, the stochastic parallel thermal transport is significantly reduced when compared to the standard analytic model [A. B. Rechester and M.N. Rosenbluth, Phys. Rev. Lett. 40, 38 (1978)] and the non-axisymmetric perpendicular radial particle transport is significantly enhanced from the axisymmetric level. These trends agree with recent experimental result trends [T. E. Evans, R. A. Moyer, K. H. Burrell, M. E. Fenstermacher, I. Joseph, A. W. Leonard, T. H. Osborne, G. D. Porter, M. J. Schaffer, P. B. Snyder, P. R. Thomas, J. G. Watkins, and W. P. West, Nature Phys. 2, 419 (2006)]. It is also found, as a side product, that an artificial local reduction of the vacuum RMP fields in the vicinity of the magnetic separatrix can bring the kinetic simulation results to a more detailed agreement with experimental plasma profiles.
\end{abstract}

PACS numbers: 28.52.-s, 52.55.Fa, 52.55.Dy

*gypark@cims . nyu .edu

†cschang@cims. nyu. edu 


\section{INTRODUCTION}

Charged particle dynamics in a stochastic magnetic field is an important research topic in many areas. In this work, we report a new theoretical understanding at first-principles level of as yet unexplained, but significant, experimental observations on tokamak plasmas [1-3], which may point the way for ITER[4] to achieve essential elements of its required configuration. A successful ITER experiment is expected to demand a high edge plasma pressure in the form of so-called H-mode pedestal (see Fig. 1). However, high edge pedestal requires a steep edge pressure gradient $\nabla p$, which destabilizes large scale edge localized modes (ELMs). Theoretically, ELMs can be avoided if the average $\nabla p$ is reduced by widening the steep gradient region. One such technique is to create a localized stochastic magnetic field by applying small resonant magnetic perturbations (RMPs) using external coils [5].

Experiments on the DIII-D tokamak [1-3, 6], and later on JET (Joint European Torus) [7], have indeed demonstrated ELM control using RMPs, but $\nabla p$ changes are achieved in an unexpected manner: After applying RMPs, experiments i) do not observe a complete collapse of the electron temperature $T_{e}$, opposite to the prediction by the standard plasma transport theory in a stochastic magnetic field by Rechester and Rosenbluth (R-R theory) [8], and ii) do observe a strong reduction of the pedestal electron density $n_{e}$. Experiments also observe that a significantly enhanced particle transport deep in the plasma, either by unscreened magnetic field stochasticity or by RMP-induced turbulenct transport, is a necessary condition for a successful widening of the steep edge $\nabla p$ region [9]. Figure 1 displays the plasma profiles for DIII-D discharge 126006 versus the poloidal magnetic flux $\psi_{N}$, a radial coordinate normalized to be 0 at the magnetic axis and 1 at the magnetic separatrix. Analytic and fluid modelings have used the baseline ingredients of R-R theory for the electron heat transport in stochastic magnetic field with only incomplete understanding of the plasma transport mechanisms in stochastic magnetic field [10-14]. Incomplete understanding of the plasma transport mechanisms in stochastic magnetic field in toroidal confinement geometry makes the ELM control by RMP coils in future tokamak reactors uncertain. Here, first-principles-level kinetic simulation in a realistic magnetic separatrix geometry, with electrons and ions orbiting under $3 \mathrm{D}$ vacuum RMPs, self-consistent radial electric field $E_{r}$ and Coulomb/neutral collisions, are presented for the first time which shows that electron heat transport is significantly reduced from the R-R theory predictions and the particle flux is significantly enhanced from the 


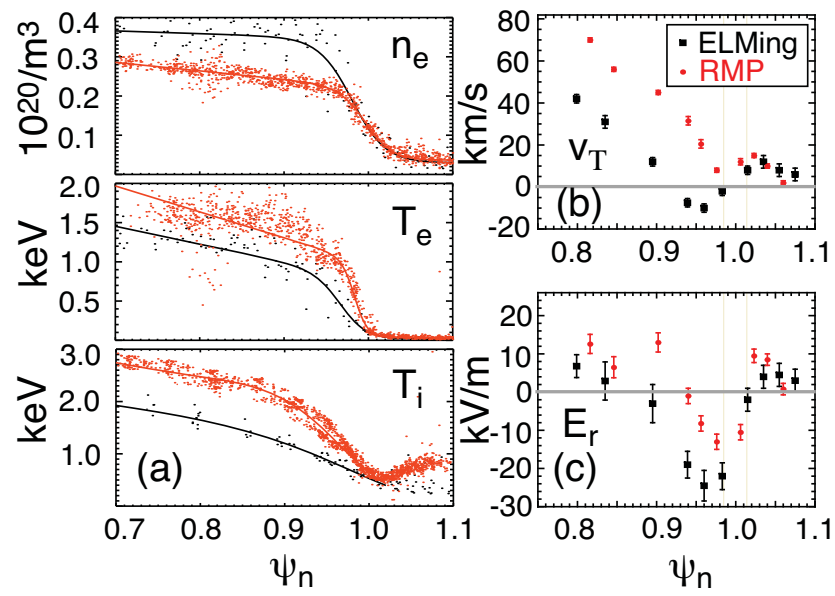

FIG. 1: (Color) Experimentally measured edge profiles along the outside midplane on DIII-D before (black) and after (red) the RMP is turned on: (a) $n_{e}, T_{e}, T_{i}$; (b) toroidal carbon rotation $V_{T}$; and (c) $E_{r}$. In the SOL, $\psi_{N}>1, T_{i}$ may be inaccurate due to a non-Maxwellian tail.

axisymmetric neoclassical level after $4 \mathrm{~ms}$ of the RPM application.

In this work, we use the kinetic ion-electron-neutral guiding-center code XGC0 [15, 16], which includes the particle source from wall-recycling and neutral Monte Carlo transport, the heat and momentum fluxes from the core plasma, the electron cooling from impurity radiation, and the plasma losses to the material wall. The full distribution function (as opposed to the perturbed distritution in the so-called delta-f method) is calculated in order to account for wall losses, neutral ionization and profile evolution. Under fixed externally applied RMP fields, the present study includes most of the relevant transport physics selfconsistently, but assumes that the electrostatic potential $\Phi$ variations within a flux surface and the RMP-induced changes to turbulence transport are negligible. Plasma screening is suspected to play an important role in the actual RMP distribution, but is difficult to calculate [17-21]. Numerical simulation of plasma screened RMPs, self-consistently with kinetic plasma dynamics, electric field response and plasma rotation, is currently under invetigation and will be a subject of a future report.

\section{A MODEL DIII-D PLASMA AND THE SIMULATION PROCEDURE}

The simulations of DIII-D discharge 126006, an RMP ELM-control experiment with plasma current of $1.52 \mathrm{MA}$, major radius of $1.76 \mathrm{~m}$, outboard minor radius $0.57 \mathrm{~m}$, and 
toroidal field $B_{0}=1.88 T$ are presented in this report. The realistic diverted tokamak equilibrium and wall geometry are imported from EFIT data [22]. Ion and electron trajectories follow the 5D Lagrangian guiding-center equations of motion [23] in the 3D magnetic field including the weak vacuum RMPs, and in the $1 \mathrm{D} \Phi\left(\psi_{N}\right)$ which is calculated as function of unperturbed magnetic surface label $\psi_{N}$. Self-consistency of $E_{r}=-\nabla \psi_{N} \partial \Phi\left(\psi_{N}\right) / \partial \psi_{N}$ is a crucial feature. In SOL, the sheath potential is obtained from a logical sheath method extended from Ref. [24], requiring that the sum of parallel and perpendicular (to $\vec{B}$-field) charge losses vanishes.

$\tilde{B}$ in tokamak geometry without a plasma response is calculated using the TRIP3D code [25], from the DIII-D I-coils carrying $4 \mathrm{kAt}$ [1]. Field errors are assumed to be perfectly corrected by the correction coils. Certain toroidal $n$ and poloidal $m$ Fourier harmonics of the field perturbation $\tilde{B}_{n m}$ are resonant with the local field line pitch near the plasma edge and cause field lines to become stochastic. The pitch-resonant components have the magnitude $\tilde{B}_{n m} / B_{0} \simeq 3.2-3.7 \times 10^{-4}$.

In $\mathrm{XGC0}, 3 \mathrm{eV}$ neutral $\mathrm{D}$ atoms are born near the material wall (at $\psi_{N}=1.04$ ), with the recycling coefficient 0.9 to represent a strong out-pumping of the neutral gas in the experiment. Approximate experimental levels of power (6 MW, equally divided between electrons and ions) and toroidal torque ( $4 \mathrm{~N}-\mathrm{m}$, from a neutral beam) are added at the coreedge boundary of the simulation domain. Monte-Carlo Coulomb collisions conserve particle number, momentum and energy. Neutral and plasma particles undergo charge exchange and ionization collisions with the Maxwellian background component of each other. Electron cooling due to carbon impurity radiation is included using ADPAK data [26]. Carbon density relative to $n_{e}$ in SOL is assumed to be $10 \%$ and negligible in the closed magnetic surface region.

The simulation procedure is as follows: First, the axisymmetric H-mode profile plasma is simulated before the RMP is turned on. The edge $E_{r}$ profile is established very rapidly, on the ion banana orbit time scale $(\sim 0.1 \mathrm{~ms})$ and the pedestal plasma reaches a "quasi-steady" state at the new $E_{r}$ profile, but still evolves on the pedestal transport time scale. Here, the "quasi-steady" state is defined to have steady $E_{r}$ while the plasma profile is evolving in transport time scale. Afterward, the RMP field is turned on. The new RMP-driven $E_{r}$ profile is established rapidly again to yield a new "quasi-steady" state which is significantly different from the non-RMP state. The simulation duration after the RMPs are turned 
on is about $4 \mathrm{~ms}$, which is long enough to establish a new RMP-driven $E_{r}$ profile. The plasma still evolves on the RMP-drven transport time scale: the density decreases and the temperature increases. A steady state plasma profile has not been established yet by the end of the simulation duration. Thus, the transport analysis presented cannot be claimed to be the same as that of steady state plasma, which, in experiment, is established a long time after the simulation duration.

Only a small amount $\left(D=\chi_{i}=\chi_{e}=0.1 \mathrm{~m}^{2} / \mathrm{s}\right)$ of anomalous radial transport is necessary to generate $n_{e}, T_{i}$ and $T_{e}$ that are close to experimental profiles before the RMP is turned on. This indicates the level of experimental anomalous transport level in the quiescent H-mode edge. In order to implement this transport, an ambipolar radial random-walk is superposed upon the Lagrangian particle trajectories. This level of anomalous transport is negligible compared to (hence, does not mask the analysis of) the RMP-driven transport. In the future, the turbulent transport information will be imported from the XGC1 gyrokinetic edge turbulence code [27].

\section{PEDESTAL RESPONSE TO THE STOCHASTIC VACUUM RMPS AND THE UNDERLYING PLASMA TRANSPORT}

Figures 2(a) and (b) show $n_{e}$ and $T_{e}$ profiles from XGC0, $0 \mathrm{~ms}$ before and $4 \mathrm{~ms}$ after the vacuum RMP is turned on. It can be seen that the pedestal $n_{e}$ has been significantly reduced by the introduction of RMPs. At the time of observation $n_{e}$ profile has not reached a steady state, but decreases at the rate given by the loss rate and the ionization rate. The radial particle flow speed across the separatrix in RMPs is $\sim 20 \mathrm{~m} / \mathrm{sec}$, somewhat greater than but roughly comparable to experimental observation [28]. Although $\nabla T_{e}$ is reduced, the pedestal $T_{e}$ has not completely collapsed (more than $3 \mathrm{MW}$ of electron heating power would yield greater $\nabla T_{e}$ and $T_{e}$ than what are shown here). These results oppose the stochastic plasma transport model of R-R and are closer to experimental trends on DIII-D. In Fig. 2(c), the pedestal $T_{i}$ correctly increases without increasing its overall gradient as the pedestal $n_{e}$ decreases. In Fig. 2(d), the ion toroidal rotation speed $V_{T}$ also shows a correct trend: an increase in the co-current direction.

Particle flux: Figure 2(f) shows various components of the quasi-steady radial ion particle flux profiles obtained from the simulation in vacuum RMPs. Total radial particle flux (black) 

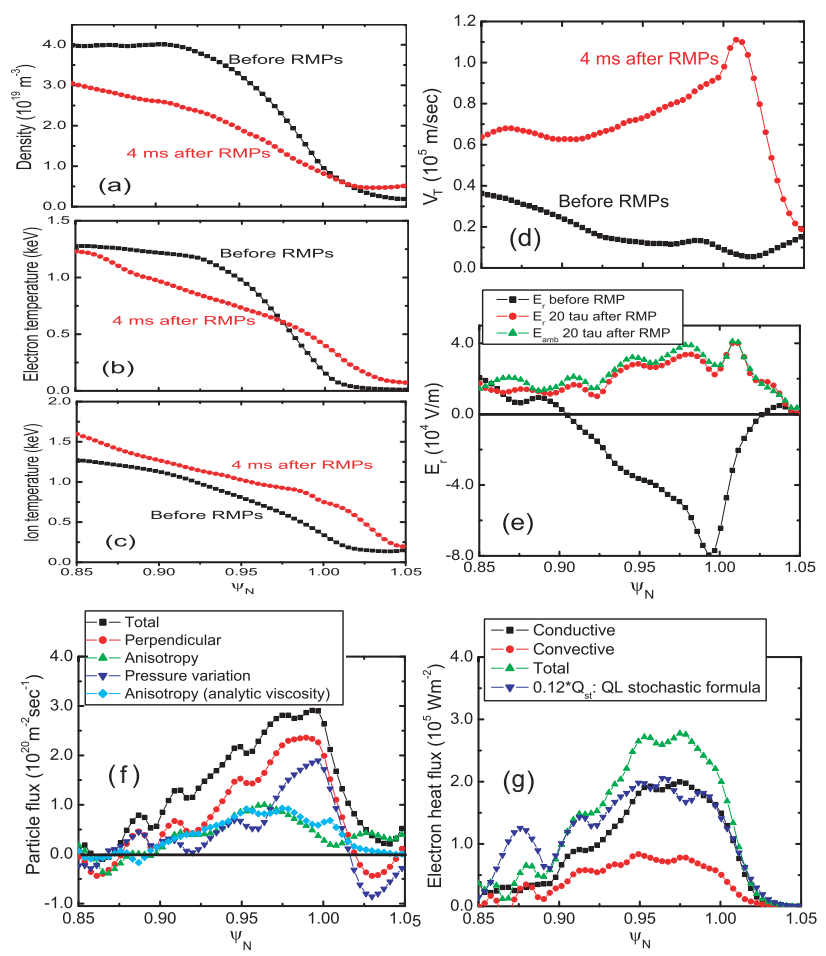

FIG. 2: (Color) Simulated profiles before (black) and $4 \mathrm{~ms}$ after (red) the vacuum RMP is turned on: (a) $n_{e}$, (b) $T_{e}$, (c) $T_{i}$, (d) $V_{T}$, and (e) $E_{r}$. Components of the radial fluxes during the RMP: (f) particle fluxes and (g) electron heat fluxes.

is dominated by perpendicular neoclassical flux (red). Parallel ion loss along $\tilde{B}$ (given by the difference between the black and red curves) is small compared to the perpendicular neoclassical loss. It is not possible for fluid models $[12,13,18,21]$ to calculate these kinetic phenomena a priori. The neoclassical perpendicular loss components can be decomposed into [29]

$$
\langle\vec{\Gamma} \cdot \nabla \psi\rangle=-\frac{I}{e}\left\langle\frac{\nabla_{\|} P_{\|}}{B}\right\rangle+\frac{I}{e}\left\langle\frac{\left(P_{\|}-P_{\perp}\right) \nabla_{\|} B}{B^{2}}\right\rangle,
$$

where the first term is from poloidal pressure variation (dark blue in Fig. 2f) and the second term is from pressure anisotropy $P_{\|}-P_{\perp}$ (green). Here $\langle\cdots\rangle$ is the flux surface average and $I=R B_{T}$. The axisymmetric neoclassical particle flux before the RMP is turned on is negligibly small $\left(<10^{18} \mathrm{~m}^{-2} \mathrm{~s}^{-1}\right)$ and is automatically ambipolar.

Strong enhancement of the particle fluxes in Fig. 2 (f) over the axisymmetric value is attributed to the RMP-driven deviation $\Delta E_{r}=E_{r}-E_{r 0}$ from the axisymmetric $E_{r 0}$. The deviation $\Delta E_{r}$ is generated to maintain ambipolarity in the nonaxisymmetric $\tilde{B}$. Trapped particles change their toroidal drift speed in response to $\Delta E_{r}$, but the passing particles do 
not. This causes a toroidal friction force $F_{T}$ to appear between the two classes of particles that is proportional to $\Delta E_{r}$. $F_{T}$ is the driver of neoclassical radial transport through $F_{T} \times B$ motion. Both the pressure-anisotropy and the parallel-variation driven particle fluxes are increased. Figure 2 (f) also shows the pressure-anisotropy driven flux using the analytic formula $\propto m n \nu_{i i} \Delta E_{r} / B_{\theta}^{2}$ for core plasmas from Ref. [30] (light blue), and shows reasonable agreement with the simulation (green) except very near the magnetic separatrix where the validity of Ref. [30] breaks down. References [11, 14] used similar analytic neoclassical formulas to explain the particle pump-out effect in RMPs.

Quasi-neutrality of the plasma is maintained by the electron particle transport along the stochastic magnetic field $\tilde{B}$, in accordance with the ion perpendicular transport. Parallel electron mobility in the radial direction along $\tilde{B}_{r} / B$, as usual, is adjusted by $E_{r}$ to yield the self-consistent $E_{r}$ and the ion/electron losses.

Heat flux: Figure 2(g) plots the conductive (black) and convective (red) decomposition of $q_{e}$ under vacuum RMPs from XGC0. The conductive part from the simulation is roughly an order of magnitude smaller ( $\simeq 1 / 8$ in the case shown) than the $\mathrm{R}-\mathrm{R}$ prediction (dark blue) with the reductution effect by the radial electric field included [10]. The convective part is non-negligible compared to the conductive part, unlike the model used in the R-R theory. We find that the electron heat conduction predicted by R-R theory is not applicable to the toroidal edge plasma mainly because majority $(\sim 70 \%)$ of the particles are magnetically trapped. Trapped electrons are reflected before traversing a decorrelation length, hence far less susceptible to the stochastic $\tilde{B}$ diffusion [31]. Even for the passing electrons, the perpendicular $E \times B$ and $\nabla B$ drifts do not permit the exact tracing of the fine scale stochastic fields (this effect, though, is found to be much weaker than the magnetic trapping effect). For ions, $q_{i}$ is about $1 / 3$ of the total $q_{e}$ and dominatly convective at the observation time (during which the particle density is being pumped out), carried through the perpendicular particle transport.

Toroidal rotation: The toroidal flow $V_{T}$ is modified by the strong transient $\overrightarrow{J_{r}} \times \vec{B}$ ion torque by the RMP onset. As $E_{r}$ is generated and ambipolarity is restored, the transient torque becomes weaker but a residual part persists since the perpendicular $J_{r}$ remains to cancel the electron $J_{\|}$loss in the stochastic magnetic field. The final flow pattern is determined by a balance between the torque source, the residual $\vec{J}_{r} \times \vec{B}$ torque, the wall and neutral drags, and the toroidal viscous damping torque in the non-axisymmetric magnetic 

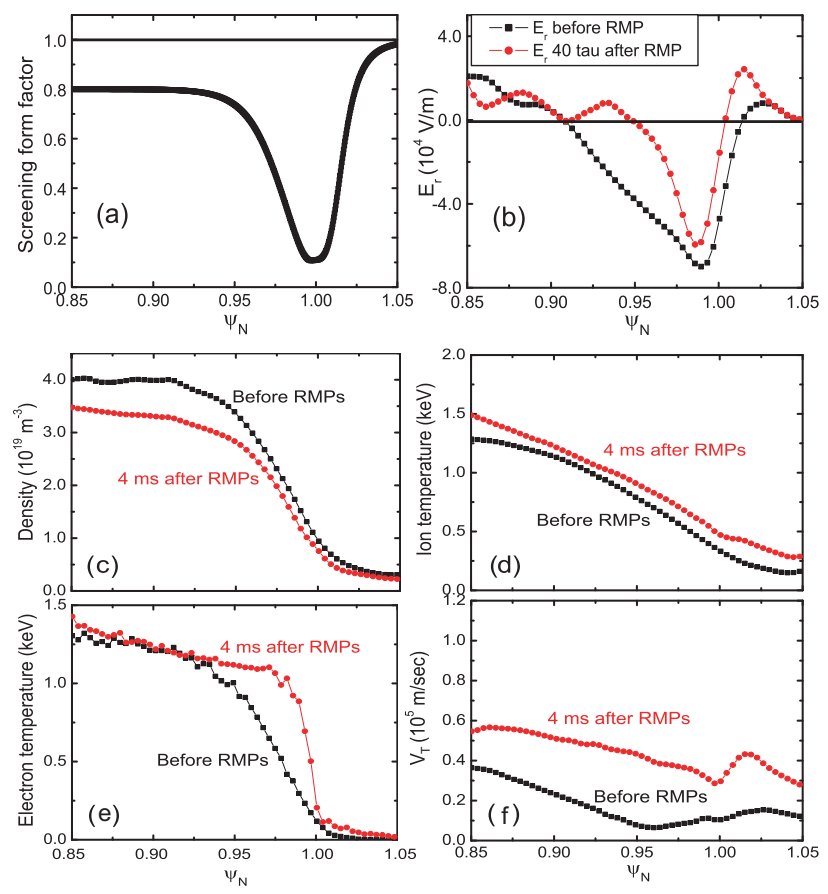

FIG. 3: The plasma profiles before (black) and $4 \mathrm{~ms}$ after (red) the screened RMP is turned on: (a) the screening factor $S\left(\psi_{N}\right)$, (b) $E_{r}$, (c) $n_{e}$, (d) $T_{e}$, (e) $T_{i}$, and (f) $V_{T}$.

field.

\section{A SIDE PRODUCT: CAN A SIMPLE RADIAL SCREENING REPRODUCE DETAILS OF THE EXPERIMENTAL OBSERVATIONS?}

Although correct trends in $n_{e}, T_{e}$, and $V_{T}$ were reproduced using the vacuum RMP model, details in $T_{e}$, and $V_{T}$ profiles, and the correct $E_{r}$ profile were not captured near the magnetic separatrix. Experimentally, after the RMP is turned on, the radial $\nabla T_{e}$ profile steepens in a narrow layer just inside the magnetic separatrix (Fig. 1a) and the negative $E_{r}$ well persists (Fig. 1c). The main cause for formation of the negative $E_{r}$ well in the axisymmetric case is known to be a consequence of the ion orbit losses in the presence of an X-point [16]. According to this process, in the presence of the stochastic magnetic field the ion orbit losses must exceed the parallel electron losses in order for the negative $E_{r}$ well to persist. Another important difference is the sharp peak in the plasma toroidal rotation profile around the magnetic separatrix (Fig. 2d), while the experiment only shows a mild increase with a dip (Fig. 1b). Similar peaking appears in the perpendicular rotation profile (not shown). 
We notice here that these discrepancies between the kinetic simulation results in the vacuum RMPs and the experimental details are localized near the magnetic separatrix. Reducing the RMPs near the separatrix would create an electron transport barrier, maintain the $E_{r}$ well, reduce the parallel electron heat transport, and reduce the $J_{r} \times B$ accelaration of toroidal flow. At the same time, if the RMPs are unaffected at the top of the pedestal and the screening is incomplete, the enhanced perpendicular transport can still explain the particle losses from inside the pedestal top as observed in the RMP experiments.

Based upon these speculations, we perform additional simulations using a simple ad hoc reduction model that lowers the vacuum RMPs by a radial form-factor $S\left(\psi_{N}\right)$ [Fig. 3(a)] near the separatrix where simulation with the vacuum RMPs was producing localized discrepancies with the details of the experimental observation. The screened RMP field is specified in a divergence-free manner. For simplicity, the reduction factor is applied to the entire RMP components, even though the screening physics may affect the resonant components only [17]. We also note here that screening of the non-resonant components may reduce the non-axisymmetric neoclassical viscosity effect.

When the depth of the screening form factor is adjusted [Fig. 3(a)] to form an $E_{r}$-well [see Figs. 1(c) and 3(b)], the simulation simultaneously reproduces aforementioned experimental details. Near the separatrix, $\nabla T_{e}$ steepens [Fig. 3(e)] and $V_{T}$ only increases moderately [Fig. 3(f)] and also shows a dip near the separatrix [Fig 1(b)]. The pedestal density drop persits as seen in experiments. Among the screening models we tested, this simple reduction model produced the best agreement with the experimental observations. Reduction in the plasma rotation to experimentally observed level by the introduction of the simple screening form factor (which reduces both resonant and non-resonant RMP components) indicates that the non-axisymmetric neoclassical toroidal viscosity effect is not dominant over the resonant stochasticity physics effects considered here. However, it is possible that we allowed the survival of the non-reresonant component in the shielded region, the local rotation could have been reduced further in the kinetic simulation.

It is to be emphasized here that the surprising success from a simple shielding form factor used in this section is merely phenomenological. The shielding form factor multiplied to the vacuum RMPs is ad-hoc and does not represent any solution from first-principles equations as intended in the rest of the paper. It is presented here as an interesting side product of the main research, of which the purpose is to enhance basic understading of the toroidal plasma 
tranport processes in stochastic magnetic fields in realistic diverted tokamak geometry.

\section{CONCLUSION AND DISCUSSION}

A kinetic transport simulation under given vacuum RMPs in realistic diveted DIII-D tokamak geometry has been performed for the first $4 \mathrm{~ms}$ after the RMP application, for the first time, with self-consistent radial electric field and plasma rotation. It is found that the stochastic parallel thermal transport is significantly reduced, compared to the prediction from the standard Rechester-Rosenbluth model [8], and that the non-axisymmetric perpendicular radial particle transport is significantly enhanced from the axisymmetric level, by toroidal kinetic neoclassical effects (magnetic trapping and ambipolar radial electric field, mostly). These trends agree with recent experimental results [1-3]. It is also found, as a side product, that a local reduction of the vacuum RMP fields in the vicinity of the magnetic separatrix can bring the kinetic simulation results to a more detailed agreement with experimental plasma profiles.

The purpose of the present study is to enhance the understading in the basic tranport physics of the toroloidal plasma in stochastic magnetic field in realistic diverted tokamak geometry, beyond the simple standard model of Rechester-Rosenbluth which neglected the neoclassical effects. Plasma response to the external RMP perturbation is not a subject of the present report. Present study indicates that the self-consitent kinetic plasma responses in a realistic geometry (including parallel current, radial electric field, plasma rotation, trapped particle effect, and density and temperature gradients) are essential to the understanding of the self-consistent RMP penetration physics. Such an investigation is currently under persuit by the authors, and will be a subject of a future publication. We note here that a low aspect ratio tokamak edge can show a different kinetic RMP-driven transport behavior from what is presented here since it has much higher fraction of the magnetically trapped particles.

\section{Acknowledgments}

We acknowledge helpful discussions with S. Ku, T. Evans, L. Horton, R. Maingi, and H. Weitzner. This work is supported by US DOE under the grant and contract DE-FG02- 
[1] T. E. Evans, R. A. Moyer, P. R. Thomas, J. G. Watkins, T. H. Osborne, J. A. Boedo, E. J. Doyle, M. E. Fenstermacher, K. H. Finken, R. J. Groebner, M. Groth, J. H. Harris, R. J. La Haye, C. J. Lasnier, S. Masuzaki, N. Ohyabu, D. G. Pretty, T. L. Rhodes, H. Reimerdes, D. L. Rudakov, M. J. Schaffer, G. Wang, and L. Zeng, Phys. Rev. Lett. 92, 235003 (2004).

[2] K. H. Burrell, T. E. Evans, E. J. Doyle, M. E. Fenstermacher, R. J. Groebner, A. W. Leonard, R. A. Moyer, T. H. Osborne, M. J. Schaffer, P. B. Snyder, P. R. Thomas, W. P. West, J. A. Boedo, A. M. Garofalo, P. Gohil, G. L. Jackson, R. J. La Haye, C. J. Lasnier, H. Reimerdes, T. L. Rhodes, J. T. Scoville, W. M. Solomon, D. M. Thomas, G. Wang, J. G. Watkins, and L. Zeng, Plasma Phys. Controlled Fusion 47, B37 (2005)

[3] T. E. Evans, R. A. Moyer, K. H. Burrell, M. E. Fenstermacher, I. Joseph, A. W. Leonard, T. H. Osborne, G. D. Porter, M. J. Schaffer, P. B. Snyder, P. R. Thomas, J. G. Watkins, and W. P. West, Nature Phys. 2, 419 (2006)

[4] R. Aymar, V. A. Chuyanov, M. Huguet, Y. Shimomura, and ITER Joint Central Team and ITER Home Teams, Nucl. Fusion 41, 1301 (2001)

[5] Ph. Ghendrih, A. Grosman, and H. Capes, Plasma Phys. Controlled Fusion 38, 1653 (1996)

[6] J. Luxon, Nucl. Fusion 42, 614 (2002)

[7] Y. Liang, H. R. Koslowski, P. R. Thomas, E. Nardon, B. Alper, P. Andrew, Y. Andrew, G. Arnoux, Y. Baranov, M. Becoulet, M. Beurskens, T. Biewer, M. Bigi, K. Crombe, E. De La Luna, P. de Vries, W. Fundamenski, S. Gerasimov, C. Giroud, M. P. Gryaznevich, N. Hawkes, S. Hotchin, D. Howell, S. Jachmich, V. Kiptily, L. Moreira, V. Parail, S. D. Pinches, E. Rachlew, and O. Zimmermann1, Phys. Rev. Lett. 98, 265004 (2007).

[8] A. B. Rechester and M. N. Rosenbluth, Phys. Rev. Lett. 40, 38 (1978).

[9] M. E. Fenstermacher, T. E. Evans, T. H. Osborne, M. J. Schaffer, M. P. Aldan, J. S. deGrassie, P. Gohil, I. Joseph, R. A. Moyer, P. B. Snyder, R. J. Groebner, M. Jakubowski, A. W. Leonard, O. Schmitz, and the DIII-D Team, Phys. Plasmas. 15, 056122 (2008).

[10] M. Z. Tokar, T. E. Evans, A. Gupta, R. Singh, P. Kaw, and R. C. Wolf, Phys. Rev. Lett. 98, 095001 (2007).

[11] M.Z.Tokar, T. R. Singh, and B. Unterberg, Phys. Plasmas 15, 072515 (2008) 
[12] I. Joseph, T. E. Evans, A. M. Runov, M. E. Fenstermacher, M. Groth, S. V. Kasilov, C. J. Lasnier, R. A. Moyer, G. D. Porter, M. J. Schaffer, R. Schneider, and J. G. Watkins, Nucl. Fusion 48, 045009 (2008).

[13] O. Schmitz, T. E. Evans, M. E. Fenstermacher, H. Frerichs, M. W. Jakubowski, M. J. Schaffer, A. Wingen, W. P. West, N. H. Brooks, K. H. Burrell, J. S. deGrassie, Y. Feng, K. H. Finken, P. Gohil, M. Groth, I. Joseph, C. J. Lasnier, M. Lehnen, A. W. Leonard, S. Mordijck, R. A. Moyer, A. Nicolai, T. H. Osborne, D. Reiter, U. Samm, K. H. Spatschek, H. Stoschus, B. Unterberg, E. A. Unterberg, J. G. Watkins, R. Wolf, and the DIII-D and TEXTOR Teams, Plasma Phys. Controlled Fusion 50, 124029 (2008).

[14] V. Rozhansky, E. Kaveeva1, P. Molchanov1, I. Veselova1, S. Voskoboynikov, D. Coster, A. Kirk, S. Lisgo and E. Nardon, Nucl. Fuaion 50, 034005 (2010)

[15] G. Park, J. Cummings, C. S. Chang, N. Podhorszki, S. Klasky, S. Ku, A. Pankin, R. Samtaney, A. Shoshani, P. Snyder, H. Strauss, L. Sugiyama, and the CPES Team, J. Phys.: Conf. Series 78, 012087 (2007).

[16] C. S. Chang and S. Ku, Phys. Plasmas 11, 2649 (2004).

[17] R. Fitzpatrick, Phys. Plasmas 5, 3325 (1998).

[18] V. A. Izzo and I. Joseph, Nucl. Fusion 48, 115004 (2008).

[19] Martin F. Heyn, Ivan B. Ivanov, Sergei V. Kasilov, Winfried Kernbichler, Ilon Joseph, Richard A. Moyer and Alexey M. Runov, Nucl. Fusion 48, 024005 (2008).

[20] J.-K. Park, A.H. Boozer, and A.H. Glasser, Phys. Plasmas 14, 052110 (2007)

[21] E. Nardon, M. Becoulet, G. Huysmans, and O. Czarny, Phys. Plasmas 14, 092501 (2007).

$[22]$ L. L. Lao, H. St. John, R. D. Stambaugh, A. G. Kellman, and W. Pfeiffer, Nucl. Fusion 25, $1611(1985)$.

[23] R. G. Littlejohn, Phys. Fluids 28, 2015 (1985).

[24] S. E. Parker, R. J. Procassini, C. K. Birdsall, and B. I. Cohen, J. Comput. Phys. 104, 41 (1993).

[25] T. E. Evans, R. A. Moyer, and P. Monat, Phys. Plasmas 3, 4957 (2002).

[26] R. Hulse, Nuclear Technology/Fusion 3, 259 (1983).

[27] C. S. Chang, S. Ku, P. H. Diamond, Z. Lin, S. Parker, T. S. Hahm, and N. Samatova, Phys. Plasmas 16, 056108 (2009).

[28] L. Zeng, T. E. Evans, T. L. Rhodes, E. J. Doyle, L. Schmitz, G. Wang, W. A. Peebles, M. 
E. Fenstermacher, and R. A. Moyer, 21st US Transport Task Force Workshop, Boulder, CO, March 25 - 28, 2008.

[29] F. L. Hinton and R. D. Hazeltine, Rev. Mod. Phys. 48, 239 (1976).

[30] S. P. Hirshman and D. J. Sigmar, Nucl. Fusion 21, 1079 (1981).

[31] B. D. G. Chandran and S. C. Cowley, Phys. Rev. Lett. 80, 3077 (1998) 